\title{
Simple Analysis and Design of a New Leaky-Wave Directional Coupler in Hybrid Dielectric-Waveguide Printed-Circuit Technology
}

\author{
José Luis Gómez-Tornero, Member, IEEE, Sofía Martínez-López, and Alejandro Álvarez-Melcón, Member, IEEE
}

\begin{abstract}
This paper presents a new theory to analyze and design a novel leaky-wave coupler based on a hybrid dielectric-waveguide printed-circuit technology. The analysis theory is based on a combined method of moments (MoM) geometrical optics (GO) technique, which makes use of the leaky mode complex propagation constant. This MoM-GO approach allows to easily obtain the response of the coupler, and to illustratively understand the working mechanism of the proposed structure. A coupler is designed to work at $5.5 \mathrm{GHz}$. Leaky mode results are compared with three-dimensional finite-element method simulations, showing the validity and accuracy of the new theory developed. This novel directional coupler presents interesting advantages when compared to previous wave couplers.
\end{abstract}

Index Terms-Directional coupler, geometrical optics (GO), leaky waves, method of moments (MoM).

\section{INTRODUCTION}

A N ORIGINAL directional coupler, based on hybrid dielectric-waveguide printed-circuit technology, was presented in [1]. The design procedure was illustrated using three-dimensional finite-element method (FEM) simulations, and the frequency response was obtained, showing the ability to control the coupling level. In this paper, an original theory based on the leaky mode complex propagation constant is described. With this theory, the response of the coupler can be obtained in a much faster way since the formulation is totally analytical and based on the leaky mode expressions. In this way, a straightforward design can be performed following the same procedure described in [1], but avoiding three-dimensional FEM simulations (which are very time consuming for large structures, as with the coupler studied in this paper). Moreover, the proposed theory helps to better understand the coupling working mechanism inherent to this coupler, illustrating the importance of higher order effects.

The structure of the proposed leaky-wave coupler is shown in Fig. 1. Two radiating dielectric waveguides, laterally shielded

Manuscript received March 17, 2006; revised May 25, 2006. This work was supported in part under Spanish National Project ESP2001-4546-PE and Spanish National Project TEC2004-04313-C02-02-TCM and under the Regional Séneca Project 2002 PB/4/FS/02.

J. L. Gómez-Tornero and A. Álvarez-Melcón are with the Department of Information Technologies and Communications, Escuela Técnica Superior de Ingenieros de Telecomunicación, Polytechnic University of Cartagena, 30202 Cartagena, Spain (e-mail: josel.gomez@upct.es; alejandro.alvarez@upct.es).

S. Martínez-López is with the Communications and Electronic Department, École Nationale Supérieure de Telécommunications, 75014 Paris, France (e-mail: sofia.martinez@enst.fr).

Digital Object Identifier 10.1109/TMTT.2006.879773

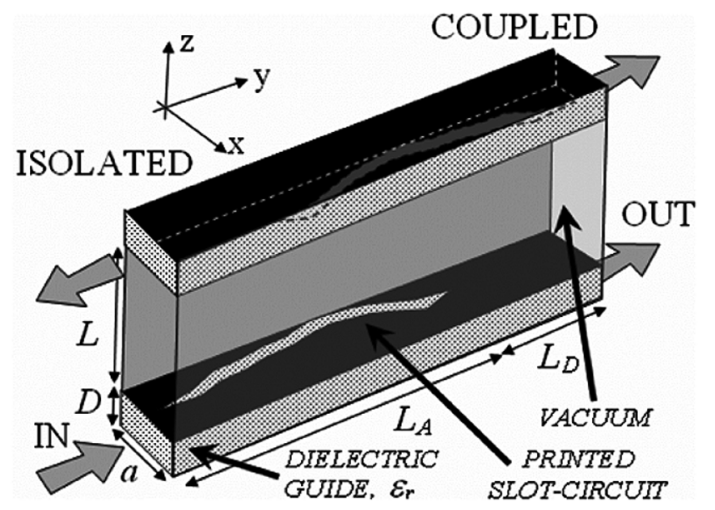

Fig. 1. Scheme of the proposed leaky-wave coupler.

by parallel plates, are faced toward each other in order to transfer energy from one to the other. Planar slot circuits are printed at the broad sides of the dielectric guides in order to excite a leaky-wave mode and to control its propagation and radiation properties. From a technological point-of-view, the proposed coupler presents a more flexible and simpler design than conventional grating couplers since the dielectric guide structure must not be mechanized to obtain a maximum efficiency design [1]. Only the printed circuit must be tapered [1]-[3]. In addition, the new coupler is based on radiation from the main space harmonic. In this way, the structure dimensions are minimized as compared to grating couplers, which are based on higher order radiating harmonics [4], [5].

\section{THEORY}

Here, the theory to analyze and design the proposed coupler is described for the first time. The working mechanism of the proposed coupler will be explained, describing the theory to analyze and design each part of the circuit in order to obtain a successful response. The theory presented in this paper is novel, and it is based on a combination of the method of moments (MoM) and geometrical optics (GO) approaches.

\section{A. MoM Analysis and Design of Dielectric Waveguides and Slot Printed Circuits}

Rectangular dielectric waveguides of width $a$, height $D$, and relative permittivity $\varepsilon_{r}$ are used to propagate the main $\mathrm{TE}_{10}$ mode. These dimensions control the $\mathrm{TE}_{10}$ mode cutoff and, therefore, determine the fast-wave frequency region where this mode can become leaky. The width of the slot, printed at the dielectric-air interface, also controls the leaky-wave region. A 


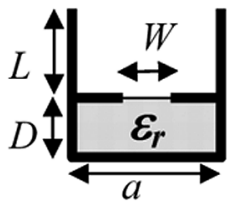

$a=22.86 \mathrm{~mm}$

$D=10.16 \mathrm{~mm}$

$\varepsilon_{r}=2.2$

$L=20 \mathrm{~mm}$

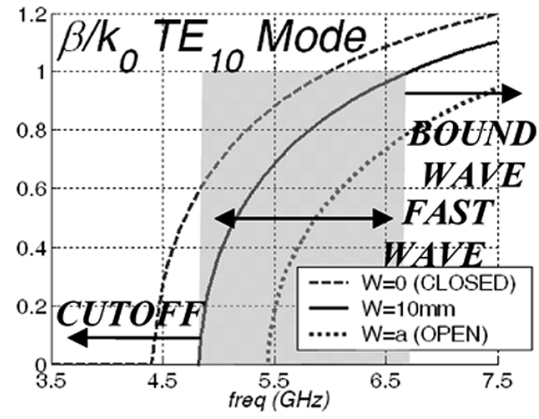

Fig. 2. Normalized phase constant of $\mathrm{TE}_{10}$ mode obtained with MoM.

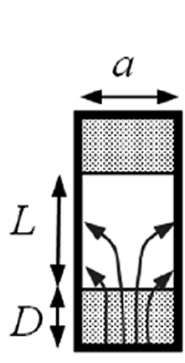

a)

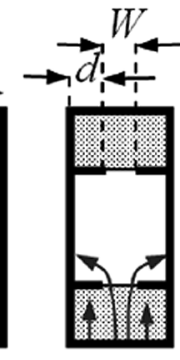

b)

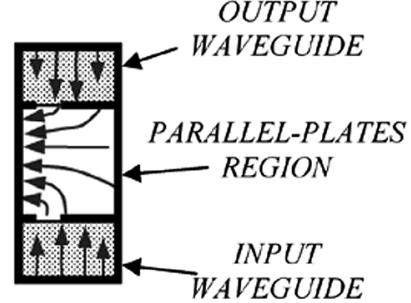

c)
Fig. 3. Asymmetry radiation and coupling mechanism.

MoM technique, especially conceived for the analysis of hybrid waveguide-planar transmission lines [2], is used to obtain the $\mathrm{TE}_{10}$ leaky mode phase and leakage constant. Fig. 2 shows the normalized phase constant $\left(\beta / k_{0}\right)$ of the $\mathrm{TE}_{10}$ mode for three different slot widths $W$ (see the inset of Fig. 2).

As it can be seen in Fig. 2, the fast wave region of the $\mathrm{TE}_{10}$ mode (dark area in Fig. 2) extends from $4.8 \mathrm{GHz}$ (cutoff region) to $6.5 \mathrm{GHz}$ (bound wave region) for a dielectric guide of dimensions $a=22.86 \mathrm{~mm}, D=10.16 \mathrm{~mm}$, and $\varepsilon_{r}=2.2$, and a centered slot of width $W=10 \mathrm{~mm}$. In addition, the slot must be uncentered with respect to the parallel plates (see Fig. 3) in order to launch the leaky wave. This asymmetry radiation mechanism is explained in detail in [3], where interesting leaky-wave antennas (LWAs) were designed using this technology. In this way, no coupling will occur between the two dielectric guides if the separation $L$ between them is large enough, and the printed slot-circuit is centered, as sketched in Fig. 3(a) and (b). However, if the slot is uncentered, there will be radiation and coupling no matter the value of $L$, as illustrated by Fig. 3(c).

To compute the coupling between the input waveguide [transmitter (TX)] and the output waveguide [receiver (RX)], an "overlapping integral" along the whole structure length can be computed, as proposed in [4] and [5]. Note that the total length is divided into $\left(L_{A}+L_{D}\right)$ according to Fig. 1, where $L_{A}$ is the radiating slot length and $L_{D}$ is the spatial offset between the TX and the RX shown in Fig. 1.

This integral computes the overlapping between the beams created by each waveguide at the reference plane located in the middle height. As sketched in Fig. 4, the emitted beams must be shaped to obtain maximum overlapping and, therefore, maximum coupling [5]. For this purpose, the printed slot circuit must be tapered along the coupler length, as explained in [3], in order

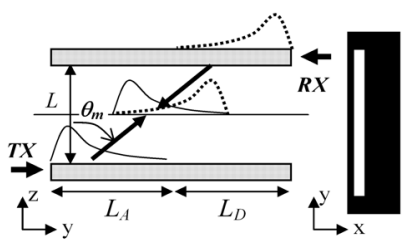

a)

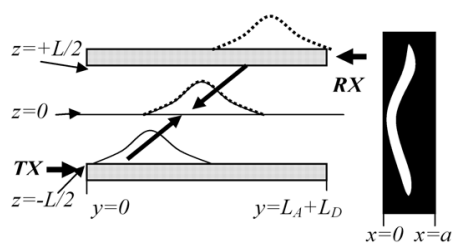

b)
Fig. 4. Necessity of tapering the printed circuit.

to obtain a cosine shaped beam [see Fig. 4(b)]. The MoM analysis approach allows to obtain the variation of the $\mathrm{TE}_{10}$ leaky mode complex longitudinal propagation constant $\left(k_{y}\right)$ along the waveguides length ( $y$-axis, see Fig. 1) for both the TX and RX couplers

$$
\begin{aligned}
& k_{y}^{\mathrm{TX}}(y)=\beta_{y}^{\mathrm{TX}}(y)-j \alpha_{y}^{\mathrm{TX}}(y) \\
& k_{y}^{\mathrm{RX}}(y)=\beta_{y}^{\mathrm{RX}}(y)-j \alpha_{y}^{\mathrm{RX}}(y) .
\end{aligned}
$$

The normalized illuminations at the TX and RX antenna apertures $\overline{M^{\mathrm{TX}}}$ and $\overline{M^{\mathrm{RX}}}$ can be computed from the following well-known equations [6]:

$$
\begin{aligned}
\overline{M^{\mathrm{TX}}}\left(y, z=-\frac{L}{2}\right) & =\frac{\sqrt{\frac{d f^{\mathrm{TX}}(y)}{d y}}}{\max \left\{\sqrt{\frac{d f^{\mathrm{TX}}(y)}{d y}}\right\}} \cdot e^{-j \beta_{y}^{\mathrm{TX}}(y)} \\
f^{\mathrm{TX}}(y) & =e^{-2 \cdot \int_{\tau=0}^{\tau=y} \alpha_{y}^{\mathrm{TX}}(\tau) \cdot d \tau} \\
\overline{M^{\mathrm{RX}}}\left(y, z=+\frac{L}{2}\right) & =\frac{\sqrt{\frac{d f^{\mathrm{RX}}(y)}{d y}}}{\max \left\{\sqrt{\frac{d f^{\mathrm{RX}}(y)}{d y}}\right\}} \cdot e^{+j \beta_{y}^{\mathrm{RX}}(y)} \\
f^{\mathrm{RX}}(y) & =e^{-2 \cdot \int_{\tau=L_{A}+L_{D}}^{\tau=y} \alpha_{y}^{\mathrm{RX}}\left(-\tau+L_{A}+L_{D}\right) \cdot d \tau} .
\end{aligned}
$$

Fig. 5(a) shows the complex illumination functions created by cosine-tapered LWAs for both the TX and RX couplers at the level of their apertures (the TX LWA is located at position $z=$ $-L / 2$, while the RX LWA is located at $z=+L / 2$, see Fig. 4). The illumination is plotted along the coupler normalized length ( $y$-axis, see Fig. 1). As can be seen in Fig. 5, cosine-shaped beams can be synthesized by tapering the printed-circuit dimensions of the LWAs. The design procedure is described in detail in [3], and it is based on the MoM analysis of the dispersion curves of the $\mathrm{TE}_{10}$ leaky mode. The TX and RX LWAs are usually designed to point at a given elevation direction, which can be computed from the leaky mode phase constant $\beta_{y}$. The TX and RX pointing angles $\theta_{\mathrm{TX}}$ and $\theta_{\mathrm{RX}}$ can be obtained from $\beta_{y}$ as [6]

$$
\begin{aligned}
& \sin \theta_{\mathrm{TX}}(y)=\frac{\beta_{y}^{\mathrm{TX}}(y)}{k_{0}} \\
& \sin \theta_{\mathrm{RX}}(y)=\frac{\beta_{y}^{\mathrm{RX}}(y)}{k_{0}}
\end{aligned}
$$

where $k_{0}$ is the free-space wavenumber in vacuum. As is illustrated in Fig. 4, the TX and RX angles should be unvarying along the antennas length in order to avoid unwanted beam dispersion. For these purposes, it can be seen from (7) and (8) that the leaky 

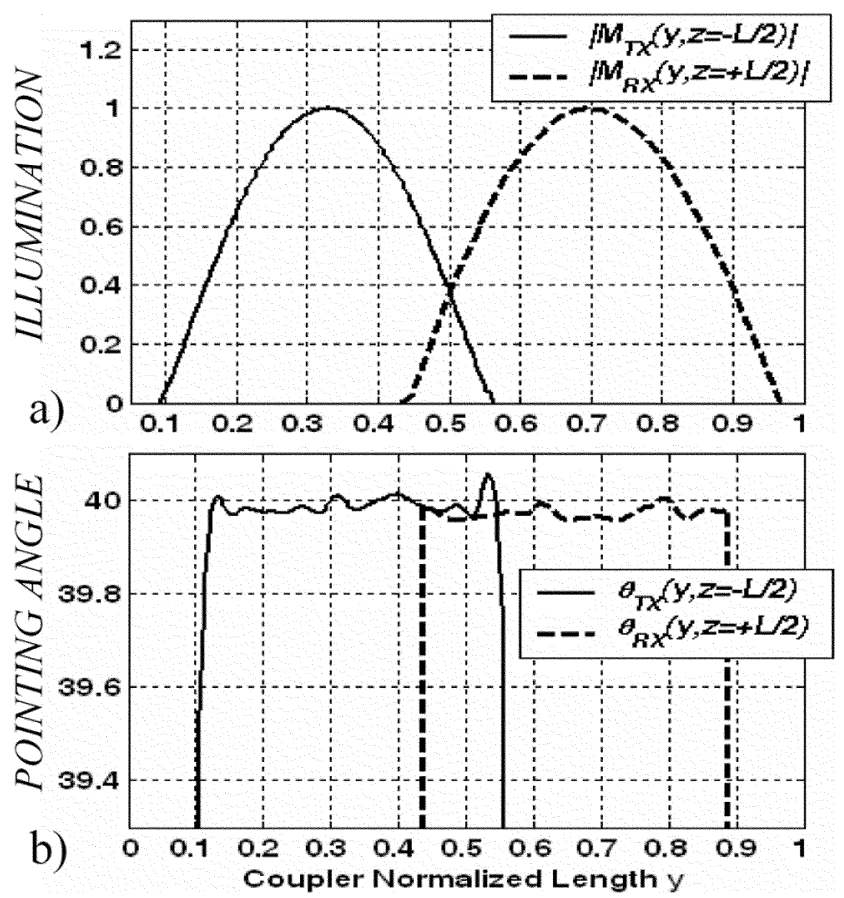

Fig. 5. Complex illumination functions for the beams created by the TX and RX LWAs at their apertures.

TABLE I

DiMENSIONS (IN MiLLIMETERS) OF THE LWAs TAPERED PRINTED SLOT CIRCUIT

\begin{tabular}{|c|c|c|c|c|c|}
\hline$y(\mathrm{~mm})$ & $W$ & $d$ & $y(\mathrm{~mm})$ & $W$ & $d$ \\
\hline 0.00 & 6.22 & 10.44 & & & \\
\hline 27.27 & 6.16 & 10.44 & 300.00 & 4.85 & 9.81 \\
\hline 54.55 & 5.97 & 10.44 & 327.27 & 4.77 & 9.70 \\
\hline 81.82 & 5.77 & 10.40 & 354.55 & 4.70 & 9.60 \\
\hline 109.09 & 5.64 & 10.36 & 381.82 & 4.66 & 9.52 \\
\hline 136.36 & 5.50 & 10.31 & 409.09 & 4.67 & 9.54 \\
\hline 163.64 & 5.36 & 10.26 & 436.36 & 4.76 & 9.69 \\
\hline 190.91 & 5.26 & 10.19 & 463.64 & 4.96 & 9.95 \\
\hline 218.18 & 5.15 & 10.11 & 490.91 & 5.29 & 10.21 \\
\hline 245.45 & 5.03 & 10.02 & 518.18 & 5.73 & 10.39 \\
\hline 272.73 & 4.94 & 9.92 & 545.45 & 6.22 & 10.44 \\
\hline
\end{tabular}

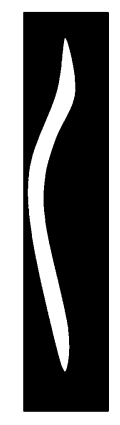

modes' phase constants should keep unchanged along the antenna aperture. Following the design procedure described in [3], a nearly constant pointing direction can be obtained for all the emitted beam length, as shown in Fig. 5(b).

Table I represents the dimensions of the printed-slot circuit obtained in the design of the TX and RX LWAs. Both antennas are identical, but the RX LWA must be located facing the TX LWA, at a height $L$ and an offset $L_{D}$, as illustrated in Figs. 1 and 4. As can be seen in Table I, the width $W$ and the position $d$ of the slot [see Fig. 3(b)] must be tapered along the waveguide length to obtain the constant-pointing cosine-shaped beams. The frequency of design is $5.5 \mathrm{GHz}$, and the length of the LWAs is $L_{A}=10 \lambda_{0}=545.45 \mathrm{~mm}$.

\section{B. GO Analysis}

The beam created at the LWAs apertures is able to travel along the parallel-plates stub of height $L$, as shown in Fig. 4 . The transverse plane located at $z=0$ will be used as the reference plane to compute the coupling coefficients. For this purpose, a simple GO model [7] is applied. Due to the fact that a uniform propagation direction is obtained for all the length of the synthesized beams $[\theta(y)$ is almost constant in Fig. 5(b)], a simple displacement can be used to obtain the illuminations created by the TX and $\mathrm{RX}$ antennas at the reference plane $(z=0)$

$$
\begin{array}{r}
\delta_{\mathrm{TX}}=\frac{L}{2} \tan \left(\overline{\theta_{\mathrm{TX}}}\right) \delta_{\mathrm{RX}}=\frac{L}{2} \tan \left(\overline{\theta_{\mathrm{RX}}}\right) \\
\overline{M^{\mathrm{TX}}}(y, z=0)=\overline{M^{\mathrm{TX}}}\left(y-\delta_{\mathrm{TX}}, z=-\frac{L}{2}\right) \\
\overline{M^{\mathrm{RX}}}(y, z=0)=\overline{M^{\mathrm{RX}}}\left(y+\delta_{\mathrm{RX}}, z=+\frac{L}{2}\right)
\end{array}
$$

where $\overline{\theta_{\mathrm{TX}}}$ and $\overline{\theta_{\mathrm{RX}}}$ are the mean values for the beam pointing direction of the TX and RX LWAs, respectively [see Fig. 5(b)]. Once the beams complex illuminations have been computed at the reference plane $(z=0)$, a normalized overlapping integral can be used to compute the coupling between these two beams along all the coupler length $\left(L_{A}+L_{D}\right)$ [5], shown in (12) at the bottom of this page.

In order to have high coupling coefficient $C_{1}$, the beams must match in the same position with the same amplitude function and with conjugated phases. This will depend on the amplitude and phases of the emitted beams $\left(\overline{M^{\mathrm{TX}}}\right.$ and $\left.\overline{M^{\mathrm{RX}}}\right)$, but it will also depend on the height of the coupler $(L)$, the length of the radiating aperture $\left(L_{A}\right)$, and the offset between the LWAs $\left(L_{D}\right)$. Fig. 6 illustrates different scenarios. The first-order coupling computed by $C_{1}$ occurs when the TX and RX beams overlap, as sketched in Fig. 6(c). A wrong shift of the beams will decrease the overlapping, as shown in Fig. 6(b) and (d).

The energy of the TX, which is not coupled to the RX, will be reflected back from the RX waveguide to the TX waveguide. In Fig. 6(a), a higher order coupling scenario is sketched. The reflected beam will be retransmitted up to the RX LWA, as is shown in Fig. 6(a). In this way, a second-order coupling coefficient can be computed from the new incident beam, shown in (13) at the bottom of the following page, where the illumination

$$
C_{1}=\frac{\int_{y=0}^{y=L_{A}+L_{D}}\left\{\overline{M^{\mathrm{TX}}}(y, z=0)\right\} \cdot\left\{\overline{M^{\mathrm{RX}}}(y, z=0)\right\}^{*} \cdot d y}{\sqrt{\int_{y=0}^{y=L_{A}+L_{D}}\left|\overline{M^{\mathrm{TX}}}(y, z=0)\right|^{2} d y \cdot \int_{y=0}^{y=L_{A}+L_{D}}\left|\overline{M^{\mathrm{RX}}}(y, z=0)\right|^{2} d y}}
$$




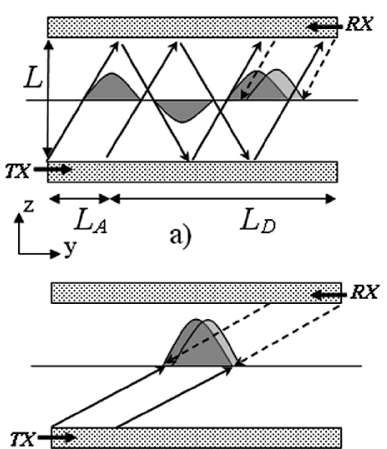

c)

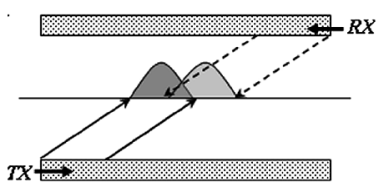

b)

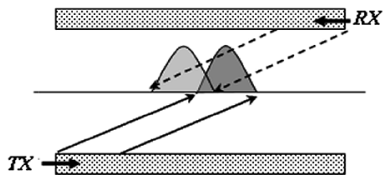

d)
Fig. 6. Four different coupling scenarios. (a) Higher order coupling. (b)-(d) Influence of beam shifting on first-order coupling.

created by the TX beam after the multiple reflections is computed using the displacement $\delta_{\mathrm{TX}}$

$$
\bar{M}_{2}^{\mathrm{TX}}(y, z=0)=\bar{M}^{\mathrm{TX}}\left(y-4 \delta_{\mathrm{TX}}, z=0\right) .
$$

This process can be recursively repeated so that the total amount of energy coupled from the TX to the RX can be computed, after multiple reflections, as

$$
C=\eta \cdot\left\{\left|C_{1}\right|+\sum_{n=2}^{N}\left[\prod_{m=1}^{n-1}\left(1-\left|C_{m}\right|\right)\right] \cdot\left|C_{n}\right|\right\} .
$$

In (15), $N$ represents the total number of reflections considered, and $\eta$ is the maximum coupling coefficient, computed from the TX and RX antennas radiation efficiencies $\eta^{\mathrm{TX}}$ and $\eta^{\mathrm{RX}}$. They are obtained from their respective leakage functions $\left(\alpha_{y}^{\mathrm{TX}}(y)\right.$ and $\left.\alpha_{y}^{\mathrm{RX}}(y)\right)$, computed in a lossless scenario and in the absence of the other antenna. The procedure for the calculation of $\alpha_{y}^{\mathrm{TX}}(y)$ and $\alpha_{y}^{\mathrm{RX}}(y)$ is detailed in [6] as follows:

$$
\begin{aligned}
\eta & =\eta^{\mathrm{TX}} \cdot \eta^{\mathrm{RX}} \\
\eta^{\mathrm{TX}} & =1-e^{-2 \cdot \int_{\tau=0}^{\tau=L_{A}} \alpha_{y}^{\mathrm{TX}}(\tau) \cdot d \tau} \\
& =1-f^{\mathrm{TX}}\left(L_{A}+L_{D}\right) \\
\eta^{\mathrm{RX}} & =1-e^{-2 \cdot \int_{\tau=L_{A}}^{\tau+L_{D}} \alpha_{y}^{\mathrm{RX}}\left(-\tau+L_{A}+L_{D}\right) \cdot d \tau} \\
& =1-f^{\mathrm{RX}}(0) .
\end{aligned}
$$

To obtain the highest coupling, the radiation efficiency of the two LWAs, $\eta^{\mathrm{TX}}$ and $\eta^{\mathrm{RX}}$ [see (16)-(18)] must be maximized. Once the leakage rate of the LWA is known (in our case, it is typically $\alpha / k_{0}=0.1$ ), the radiating slot length $L_{A}$ must be chosen to fulfill the high-efficiency requirements of a particular application [the higher $L_{A}$ is, the higher $\eta$ is obtained, see (17) and (18)]. In our case, we assure good radiation efficiency for all the designs with $L_{A}=10 \lambda_{0}\left(\eta^{\mathrm{TX}}=\eta^{\mathrm{RX}}=98 \%\right)$.

In a practical LWA coupler, the TX and RX antennas are arranged to have direct coupling (high $C_{1}$ ) at the design frequency, as shown in Fig. 6(c). Due to the frequency beam scanning response of LWA [6], the coupling decreases for frequencies below [see Fig. 6(b)] and above [see Fig. 6(d)] the center frequency. For quite low frequencies, higher order couplings can be very important, as shown in Fig. 6(a). In any case, the energy that has not been coupled after $N$ higher order couplings reaches the structure far end at $y=L_{A}+L_{D}$, where the perfect absorber must be located to avoid unwanted standing-wave phenomena. In Section III, a practical design of an LWA coupler in hybrid waveguide printed-circuit technology is performed using the previously described theory. Results obtained with a three-dimensional finite-element electromagnetic solver (FEM) will be presented to validate the theory.

\section{RESULTS}

Once the LWAs have been designed to synthesize $L_{A}=10 \lambda_{0}$ long cosine-shaped constant pointing beams (see Fig. 5), the total coupler can be analyzed and designed using the combined MoM-GO theory. As demonstrated in [1], the height of the parallel plates $(L)$ must be high enough to avoid direct coupling due to the evanescent bound wave (with $L>80 \mathrm{~mm}$, the direct coupling is below $-50 \mathrm{~dB}$ ). In this way, the coupling mechanism can only be due to the emitted leaky-wave beams.

For a given central frequency, which, in our case, is $5.5 \mathrm{GHz}$, the TX and RX slot circuits of length $L_{A}$ must be displaced at an offset $L_{D}$ [see Figs. 1 and 4(a)] in order to make the emitted beam to overlap with the trace of the receiver [5]. The theoretical value of $L_{D}$, which provides this overlapping, is given by

$$
L_{D}=L \cdot \tan \theta_{\mathrm{RAD}}
$$

where $\theta_{\mathrm{RAD}}=\theta_{\mathrm{TX}}=\theta_{\mathrm{RX}}$ [if the design is correct, as shown in Fig. 5(b)]. The radiating angle obtained from the $\mathrm{TE}_{10}$ leaky mode dispersion curve for the design frequency $(5.5 \mathrm{GHz})$ is $\theta_{\mathrm{RAD}}=40^{\circ}$ [see Fig. 5(b)] and $L$ is chosen to be $L=90 \mathrm{~mm}$. This gives rise to a theoretical offset (19) $L_{D}=75.51 \mathrm{~mm}$. Fig. 7 shows the variation of the coupling as a function of $L_{D}$ for the designed $10 \lambda_{0}$-long cosine-shaped slot-circuits, pointing at $\theta_{m}=40^{\circ}$ at $5.5 \mathrm{GHz}$. The results obtained with the leaky mode theory described in this paper are compared with a commercial FEM solver. It is checked that the maximum coupling takes place between $L_{D}=70 \mathrm{~mm}$ and $80 \mathrm{~mm}$. For other values of $L_{D}$, the traces are shifted, obtaining a smaller overlapping integral, which is translated into a lower coupling, as can be seen

$$
C_{2}=\frac{\int_{y=0}^{y=L_{A}+L_{D}}\left\{\overline{M_{2}^{\mathrm{TX}}}(y, z=0)\right\} \cdot\left\{\overline{M^{\mathrm{RX}}}(y, z=0)\right\}^{*} \cdot d y}{\sqrt{\int_{y=0}^{y=L_{A}+L_{D}}\left|\overline{M_{2}^{\mathrm{TX}}}(y, z=0)\right|^{2} d y \cdot \int_{y=0}^{y=L_{A}+L_{D}}\left|\overline{M^{\mathrm{RX}}}(y, z=0)\right|^{2} d y}}
$$




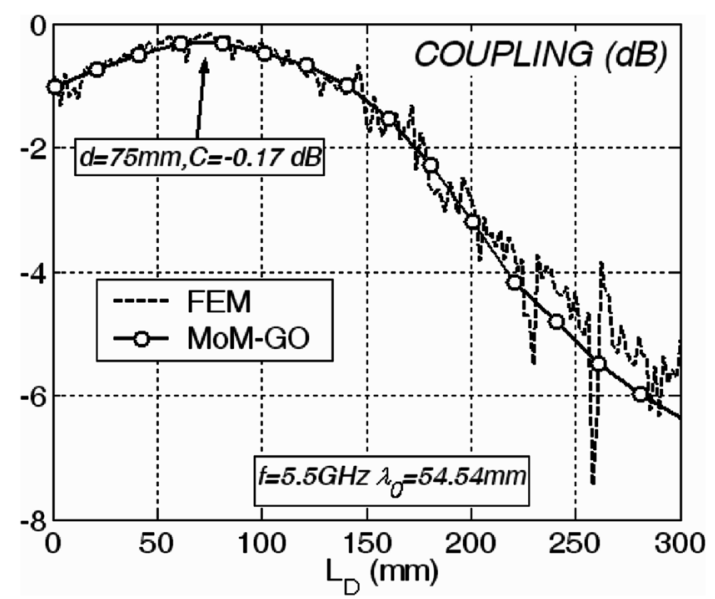

Fig. 7. Design of longitudinal offset between couplers $L_{D}(f=5.5 \mathrm{GHz}$, $L=90 \mathrm{~mm}, L_{A}=10 \lambda_{0}=545.5 \mathrm{~mm}$ ).

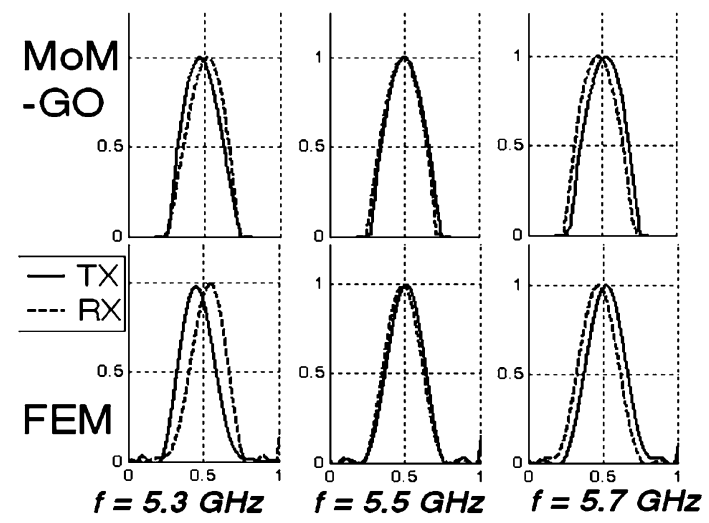

Fig. 8. Beam illumination along coupler length $(z=0)$ for different frequencies $\left(L_{D}=70 \mathrm{~mm}, L=90 \mathrm{~mm}, L_{A}=10 \lambda_{0}=545.5 \mathrm{~mm}\right)$.

in Fig. 7. Very good agreement is observed between the two different analysis approaches (leaky mode and FEM).

When frequency is varied around the design point (5.5 GHz), a beam-shifting phenomenon occurs. Due to the frequency-scanning response of LWAs [6], the beams are displaced and the overlapping is reduced. This phenomenon is illustrated in Fig. 8, where the traces created by the TX and RX LWAs at the reference level $(z=0)$ are plotted for different frequencies. The offset between the two antennas has been chosen to be $L_{D}=70 \mathrm{~mm}$. The beams obtained with the combined MoM-GO theory [see (9)-(11)] are compared to the plots obtained with FEM analysis. Very good agreement is observed for the beam shape and frequency shift.

Fig. 9 shows the dependence with frequency of the pointing direction $\theta_{\mathrm{RAD}}$ and of the radiation efficiency $\eta_{\mathrm{RAD}}$ for the designed tapered LWAs. The pointing direction $\theta_{\text {RAD }}$ is derived from the mean value applied to (7) and (8), while the radiation efficiency $\eta_{\mathrm{RAD}}$ is computed with (17) and (18). The leaky mode results are compared with FEM analysis, showing excellent agreement. As can be seen in Fig. 9, the antennas are designed to point at $\theta_{\mathrm{RAD}}=40^{\circ}$ for $f=5.5 \mathrm{GHz}$ with nearly $\eta_{\mathrm{RAD}}=100 \%$. For higher frequencies, the radiation tends to the endfire direction $\left[\theta_{\mathrm{RAD}}=90^{\circ}\right.$, see Fig. $\left.6(\mathrm{c})\right]$, while the radiation efficiency decreases [6]. For lower frequencies, the beam

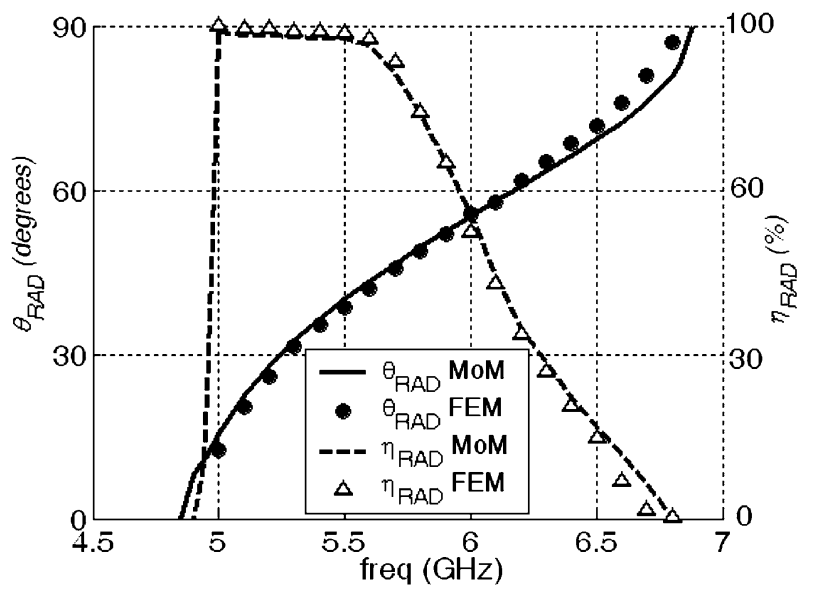

Fig. 9. LWAs pointing direction and radiation efficiency; dependence with frequency.

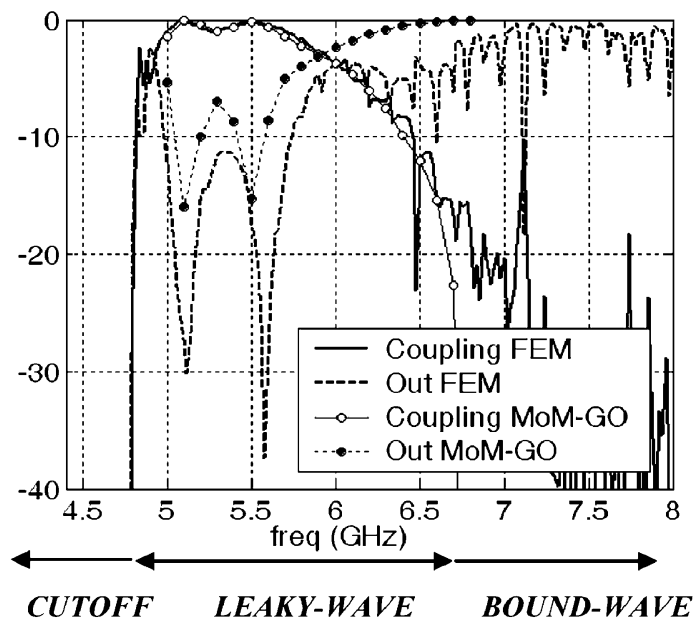

Fig. 10. Frequency response of coupling and direct output.

points closer to the broadside radiation $\left(\theta_{\mathrm{RAD}}=0^{\circ}\right)$, keeping the nearly $100 \%$ radiation efficiency. Below $4.8 \mathrm{GHz}$, the $\mathrm{TE}_{10}$ mode is at cutoff, and above $6.8 \mathrm{GHz}$, the $\mathrm{TE}_{10}$ leaky mode becomes a bound wave [6].

These three regimes can also be identified in the frequency response of the coupler shown in Fig. 10, where both the coupling and direct output responses are shown. Coupling and direct output are very low below $4.8 \mathrm{GHz}$ due to the cutoff behavior of the $\mathrm{TE}_{10}$ mode. The leaky-wave region corresponds to the range between 4.8-6.8 GHz, where it can be seen in Fig. 10 that the maximum coupling occurs. Above $6.5 \mathrm{GHz}$, the $\mathrm{TE}_{10}$ mode becomes a bound wave, making most of the injected energy to travel to the OUT port (see Fig. 1 for the definition of the ports), therefore decreasing the coupling. In the leaky-wave band, it is observed that the maximum coupling is obtained at the design frequency $(5.5 \mathrm{GHz})$. Around this frequency, the coupling decreases due to the frequency beam-shifting phenomenon illustrated in Fig. 8. However, another maximum appears at $5.1 \mathrm{GHz}$. This maximum is created by a second-order coupling, as was described and illustrated in Fig. 6(a). For frequencies above $5.5 \mathrm{GHz}$, the emitted beams diverge [see Fig. 6(b)] and coupling decreases as frequency increases, as it is checked 


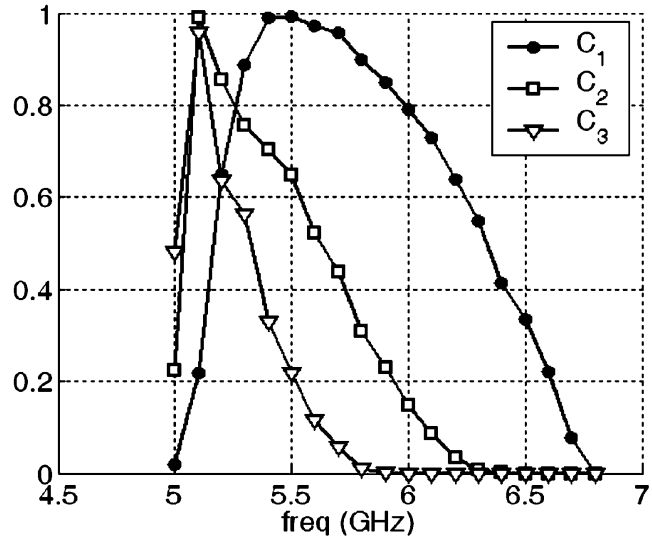

Fig. 11. Computation of overlapping factors $\left(C_{1}, C_{2}, C_{3}\right)$ as a function of frequency using the MoM-GO model.

in Fig. 10. Very good agreement is observed between the FEM analysis and the results obtained with the MoM-GO model. The total coupling was obtained from (15) using three higher order integrals. Moreover, the couplings to the OUT port were obtained assuming perfect matching and perfect isolation to the ISOLATED port (it was checked with three-dimensional FEM analysis that the return losses and isolation are below $-15 \mathrm{~dB}$ in the entire coupling band)

$$
\begin{aligned}
\text { COUPLING }(\mathrm{dB}) & =10 \log _{10}(|C|) \\
\text { OUT }(\mathrm{dB}) & =10 \log _{10}(1-|C|) .
\end{aligned}
$$

Fig. 11 shows the absolute values of the first three overlapping factors $\left(C_{1}, C_{2}\right.$, and $\left.C_{3}\right)$, computed with the MoM-GO model using (9)-(14). The first-order overlapping $\left(C_{1}\right)$ dominates at the design frequency $\left(C_{1}=1\right.$ at $\left.5.5 \mathrm{GHz}\right)$ for which there is direct sight between the TX and RX LWAs. For lower frequencies, the TX and RX beams overlap after higher order reflections due to the nearly normal angle of emission [see Fig. 6(a)]. This phenomenon is checked by the high values of $C_{2}$ and $C_{3}$ for the frequency of $5.1 \mathrm{GHz}$. As a result, another maximum of total coupling $C$ is obtained at $5.1 \mathrm{GHz}$, as can be seen in Fig. 10. It is necessary to take into account these higher order overlapping factors to accurately model the response of the designed LWA directional coupler.

Moreover, Fig. 11 helps to understand the election of the index $N$ in (15), which stands for the total number of reflections considered. As can be seen in Fig. 11, there are two significant contributions to the total coupling: the one from the direct beam $\left(C_{1}\right)$ at $5.5 \mathrm{GHz}$ and the higher order reflections $\left(C_{2}\right.$ and $\left.C_{3}\right)$ at $5.1 \mathrm{GHz}$, just after the cutoff frequency. Although the value for $C_{3}$ is quite high at $5.1 \mathrm{GHz}\left(\left|C_{3}\right| \approx 1\right)$, one can readily see that, according to (15), $C_{3}$ does not contribute much to the total response. This is due to the fact that most of the energy is already coupled to the second-order coefficient $\left|C_{2}\right|$, leaving very little energy left ( $1-\left|C_{2}\right|$ in (15) to be coupled to the third-order reflection. This phenomenon explains the results shown in Fig. 12, where the convergence of the total coupling $C(15)$ frequency response is shown as a function of the index $N$. It can be seen

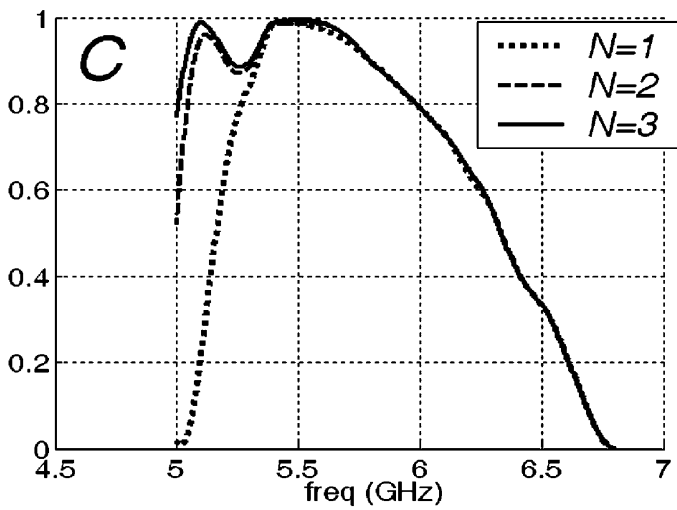

Fig. 12. Convergence of the MoM-GO coupling model with the number of significant contributions $N$.

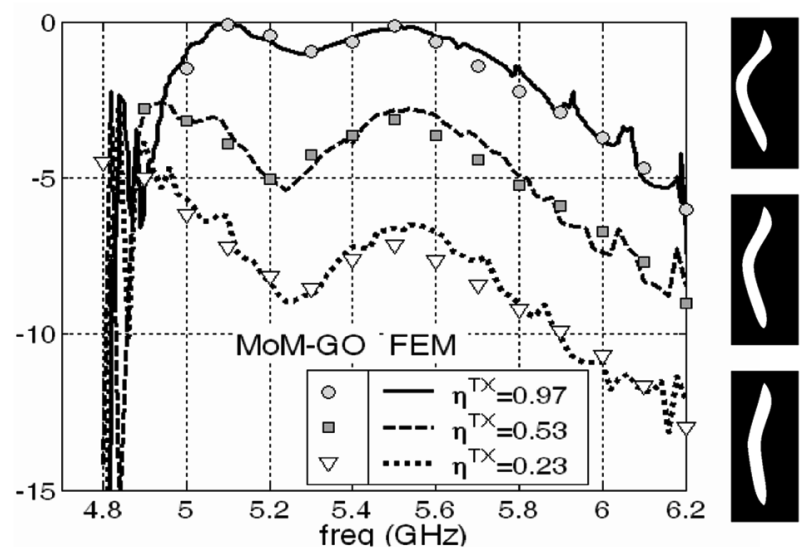

Fig. 13. Adjustment of the coupling level by varying the radiation efficiency of the TX LWA.

how with $N=2$ coefficients, the total response has almost converged. All the results shown in this paper are for $N=3$ to assure convergence.

All the previous results correspond to a directional coupler designed to obtain a coupling level of $C=-0.2 \mathrm{~dB}$ at the design frequency of $5.5 \mathrm{GHz}$. The maximum coupling level is controlled by the radiation efficiency of the TX and RX LWAs, $\eta^{\mathrm{TX}}$ and $\eta^{\mathrm{RX}}$ [see (17) and (18)]. Once the first-order overlapping integral (12) is maximized at the design frequency $\left(\left|C_{1}\right|=1\right)$, the coupling level (15) can be computed as

$$
C=\eta=\eta^{\mathrm{TX}} \cdot \eta^{\mathrm{RX}} .
$$

If the RX LWA is designed with maximum radiation efficiency $\left(\eta^{\mathrm{RX}} \approx 1\right)$, the coupling level can be determined by the TX LWA efficiency $\eta^{\mathrm{TX}}$. In this way, the input guide printed circuit will be responsible for controlling the coupling level of the entire directional coupler. Fig. 13 shows the results obtained for three couplers with maximum overlapping $C_{1}$ designed at $5.5 \mathrm{GHz}$. One coupler is designed for a 95\% coupling level $\left(\eta_{=}^{\mathrm{TX}} \eta^{\mathrm{RX}}=0.97, C=0.95=-0.2 \mathrm{~dB}\right)$, the other for $52 \%$ of coupling $\left(\eta^{\mathrm{RX}}=0.97, \eta^{\mathrm{TX}}=0.53, C=0.52=-2.8 \mathrm{~dB}\right)$, and the last one for a coupling level of $22 \%\left(\eta^{\mathrm{RX}}=0.97\right.$, $\eta^{\mathrm{TX}}=0.23, C=0.22=-6.6 \mathrm{~dB}$ ). On the right-hand side of Fig. 13, the layout of the three TX LWAs printed circuits is sketched, which is designed using the asymmetry radiation 


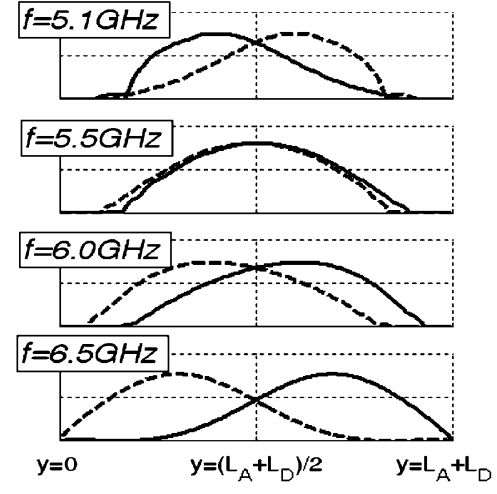

a)

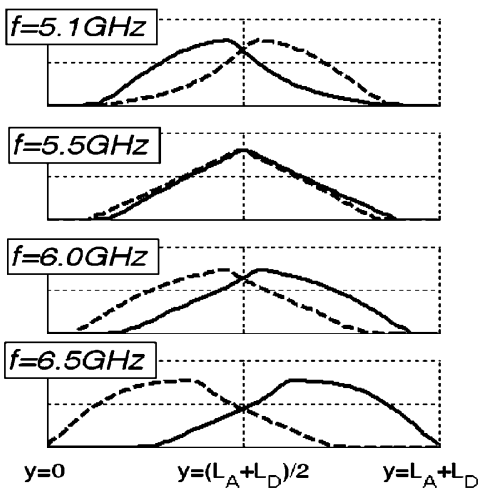

b)

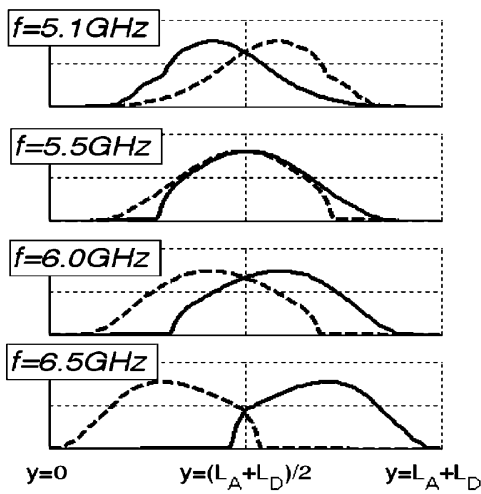

c)

Fig. 14. Illumination of TX (continuous line) and RX (dotted line) LWAs along the coupler longitudinal direction $(y)$ at the reference plane $(z=0)$ for four different frequencies in the coupling band and for three different beam shapes: (a) cosine, (b) triangular, and (c) square cosine.

principle described in [3]. To decrease the radiation efficiency of the TX LWA $\eta^{\mathrm{TX}}$, the asymmetry of the tapered slot-circuit must be decreased, as can be seen in the layouts of Fig. 13. The results obtained with the proposed MoM-GO theory are compared with FEM analysis, observing very good agreement. For all cases, it is observed the aforementioned higher order coupling at low frequencies (another maximum of coupling around $5 \mathrm{GHz})$.

Finally, a study on the influence of the slot profiles on the coupling characteristics was performed. The authors have obtained similar coupling frequency responses by using three different symmetrical beam shapes, namely, cosine-beam, triangular-beam, and square-cosine beam. Each shape is synthesized using a different tapered slot profile, as explained in [3]. The maximum coupling level (obtained at the frequency of design) does not depend on the beam shape, but on the radiation efficiency of the TX and RX LWAs [see (22)], provided the correct alignment between the two radiating waveguides is assured [see (19)].

Fig. 14 shows the beams created by the TX and RX LWAs at the reference plane $(z=0$, where the overlapping integrals are computed) for the aforementioned beam shapes and for four different frequencies of the coupling band (5.1, 5.5, 6, and $6.5 \mathrm{GHz})$. At the design frequency $(5.5 \mathrm{GHz})$, the desired beam shape is synthesized, and perfect overlapping is achieved by the correct alignment of the couplers [due to $L_{D}$, see (19)]. For other frequencies, the overlap decreases due to the beamfrequency-shifting effect, and also due to the distortion of the emitted beams. The graphs shown in Fig. 14 are obtained with the MoM-GO approach, and have been compared to FEM results, showing very good agreement.

The frequency response does not change much for these three different slot profiles, as illustrated by Fig. 15, where 95\% coupling efficiency couplers were designed using the three aforementioned beam shapes. A slight difference in the coupling frequency response is observed. This is due to the fact that the evolution of the overlapping factor with frequency varies from one beam shape to another, as can be depicted from Fig. 14. Fig. 15 shows FEM simulations, together with results from MoM-GO model for the cosine case (the results for the

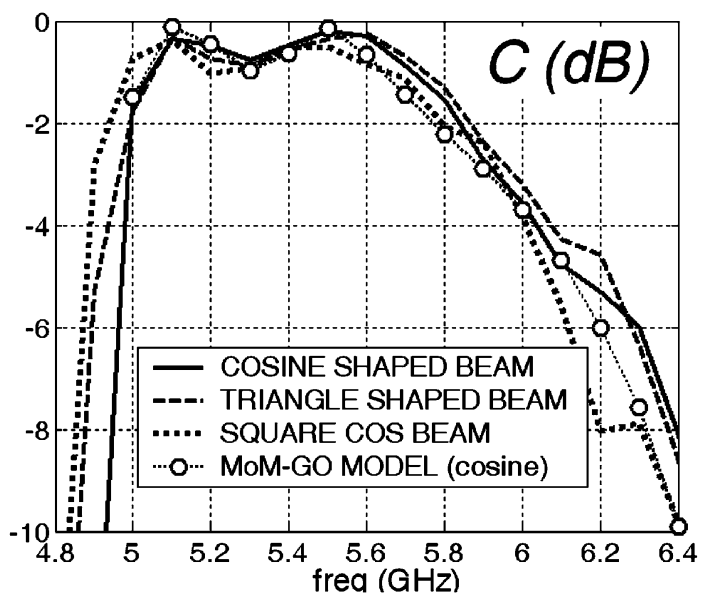

Fig. 15. Variation of the coupling frequency response for three different beam shapes.

triangle and square cosine shapes are similar, and they are not shown for the shake of clarity).

It must be emphasized that the proposed theory provides fast and accurate results for complex three-dimensional structures due to its simplicity and analytical nature. This simplicity has been achieved by splitting the analysis of the whole three-dimensional structure into several steps. First, the evolution along the $y$-axis of the leaky-wave complex propagation constants is obtained for the TX and RX radiating waveguides by means of a two-dimensional leaky mode analysis ( $x-z$-plane, see Fig. 1). The complex illumination functions along the longitudinal axis $M(y)$ are then straightforwardly derived for both the emitted and received beams. Overlapping integrals only in the $y$-direction (one-dimensional analysis) can be applied without losing accuracy. The beam coupling does not depend on the $x$-axis due to the fact that the field distribution along the $x$-direction is uniform (the radiated beam is basically a horizontally polarized oblique plane wave, as explained in [3] and [10]). A simple GO model in one dimension ( $y$-axis) can also be applied without losing accuracy due to the uniform pointing direction of the synthesized beams. Moreover, the mutual influence between the TX and RX waveguides is negligible, provided that the parallel 
plates' height is higher than $L / \lambda_{0}=1$. Under this condition, it is a good approximation to compute the normalized illumination functions considering that TX and RX are isolated.

The analysis and design of these types of couplers is much more time consuming if generic three-dimensional numerical electromagnetic solvers are used as FEM analysis. The development of a specific and simpler theory is very convenient due to the large electrical dimensions of this type of traveling-wave couplers. This is also the case for other couplers based on nonradiative dielectric (NRD) technology [8], [9]. For instance, the coupler designed in this paper has a total length $L_{A}+L_{D} \approx$ $11.5 \lambda_{0}$, a width $a \approx 0.5 \lambda_{0}$, and a total height $L+2 D \approx 2 \lambda_{0}$ (see Fig. 1).

The simple combination of the MoM and GO gives accurate results due to the particular configuration of the studied leaky-wave coupler, which is laterally shielded by metallic walls. Other authors have used other approaches to analyze other type of couplers such as the perturbation theory [4], Marcuse's coupling formula [8], or the coupled mode theory [9]. In [5], a similar overlapping integral approach was used to obtain a scattering matrix representation of a dielectric grating coupler. However, in [5], only the conditions to obtain maximum coupling at the design frequency were studied. With the proposed theory, the response of a leaky-wave coupler can be accurately obtained, for the first time, for any frequency in the leaky-wave region, using a simple GO analysis based on the dispersion of the synthesized beams.

\section{CONCLUSION}

A new theory to analyze and design a new leaky-wave directional coupler in hybrid dielectric-waveguide printed-circuit technology has been presented. A MoM approach has been used to obtain the rectangular dielectric guide dimensions and the tapered geometry of the printed slot circuit in order to control the complex propagation constant of the $\mathrm{TE}_{10}$ leaky mode. In this way, a cosine shaped beam can be synthesized to obtain maximum coupling efficiency between the input and output couplers. Using GO, the displacement of the transmitted beams as they propagate through the coupler can be computed. In this way, total coupling is obtained from the computation of the overlapping integrals in the reference plane. It has been demonstrated that the offset between the transmitter and receiver leaky-wave radiating slots must be properly chosen to maximize the overlapping at the design frequency. Results obtained using a three-dimensional electromagnetic solver based on the FEM have been compared to predictions based on this leaky-wave MoM-GO approach, showing very good agreement. It has been confirmed that it is important to take into account higher order coupling effects due to reflections in the structure. Finally, it has been shown how the printed circuit can also control the radiation efficiency of the transmitter slot, allowing for the adjustment of the coupling level. The influence of the slot profile has also been studied by comparing the coupling for three different radiated beam shapes (cosine, triangular, and square cosine). The theory developed in this paper allows to easily design these types of couplers using the information of the complex propagation constant of the radiating leaky mode. This modal approach has been validated by comparisons with full-wave three-dimensional FEM simulations.

\section{REFERENCES}

[1] J. L. Gómez, S. Martínez, D. Cañete, J. Pascual, and A. A. Melcón, "A new leaky-wave directional coupler in hybrid dielectric-waveguide printed-circuit technology," in IEEE MTT-S Int. Microw. Symp. Dig., San Francisco, CA, Jun. 11-16, 2006, pp. 1718-1721.

[2] J. L. Gómez and A. A. Melcón, "Nonorthogonality relations between complex-hybrid modes: An application for the leaky-wave analysis of laterally-shielded top-open planar transmission lines," IEEE Trans. Microw. Theory Tech., vol. 52, no. 3, pp. 760-767, Mar. 2004.

[3] J. L. Gómez, A de la Torre, D. Cañete, M. Gugliemi, and A. A. Melcón, "Design of tapered leaky-wave antennas in hybrid waveguide-planar technology for millimeter waveband applications," IEEE Trans. Antennas Propag., vol. 53, no. 8, pp. 2563-2577, Aug. 2005.

[4] T. Tamir and S. T. Peng, "Analysis and design of grating couplers," Appl. Phys., vol. 14, pp. 235-254, 1977.

[5] R. Ulrich, "Efficiency of optical-grating couplers," J. Opt. Soc. Amer., vol. 63, no. 11, pp. 1419-1431, Nov. 1973.

[6] A. A. Oliner, , R. Johnson, Ed., Leaky-Wave Antennas in Antenna Engineering Handbook, 3rd ed. New York: McGraw-Hill, 1993, ch. 10.

[7] D. A. McNamara, C. W. I. Pistorius, and J. A. G. Malherbe, Introduction to the Uniform Geometrical Theory of Diffraction, ser. Microw. Library. Norwood, MA: Artech House, 1990.

[8] T. Yoneyama, N. Tozawa, and S. Nishida, "Coupling characteristics of nonradiative dielectric waveguides," IEEE Trans. Microw. Theory Tech., vol. MTT-31, no. 8, pp. 648-654, Aug. 1983.

[9] D.-C. Niu, T. Yoneyama, and T. Itoh, "Analysis and measurement of NRD-guide leaky wave coupler in ka band," IEEE Trans. Microw. Theory Tech., vol. 41, no. 12, pp. 2126-2132, Dec. 1993.

[10] J. L. Gómez and A. A. Melcón, "Radiation analysis in the space domain of laterally-shielded planar transmission lines. Part II: Applications," Radio Sci., vol. 39, no. RS3006, pp. 1-10, Jun. 2004.

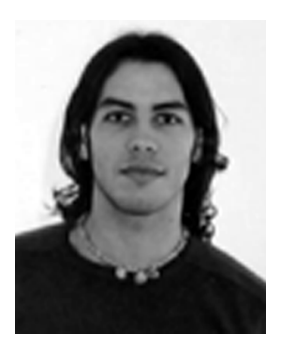

José Luis Gómez-Tornero (M'06) was born in Murcia, Spain, in 1977. He received the Telecommunications Engineer degree from the Polytechnic University of Valencia (UPV), Valencia, Spain, in 2001, and the Ph.D. degree (laurea cum laude) in telecommunication engineering from the Technical University of Cartagena (UPCT), Cartagena, Spain, in 2005.

In 1999, he joined the Radiocommunications Department, UPV, as a Research Student, where he was involved in the development of analytical and numerical tools for the study and automated design of microwave filters in waveguide technology for space applications. In 2000, he joined the Radio Frequency Division, Industry Alcatel Espacio, Madrid, Spain, where he was involved with the development of microwave active circuits for telemetry, tracking, and control (TTC) transponders implicated in many different spatial missions for the European Space Agency (ESA), National Aeronautics Space Administration (NASA) and other space agencies. In 2001, he joined the Technical University of Cartagena, as an Assistant Professor, where he currently develops his teaching activities. Since October 2005, he has been Vice Dean for students and lecture affairs with the Telecommunication Engineering Faculty, Technical University of Cartagena. His scientific research is focused on the analysis and design of leaky-wave antennas for millimeter-waveband applications and the development of numerical methods for the analysis of novel passive radiating structures in planar and waveguide technologies. His scientific interests also include the study of active devices for microwave and millimeter wavebands such as oscillators and active antennas.

Dr. Gómez-Tornero was the recipient of the 2004 Second National Award presented by the EPSON-Ibérica Foundation for the best doctoral project in the field of technology of information and communications (TIC). He was also the recipient of the 2006 Vodafone Foundation Colegio Oficial de Ingenieros de Telecomunicación (COIT/AEIT) Award presented to the best Spanish doctoral thesis in the area of advanced mobile communications technologies. 


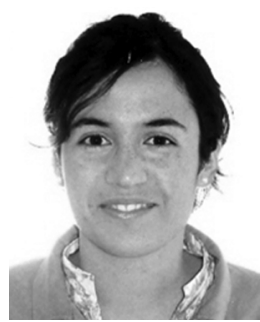

Sofía Martínez-López was born in Murcia, Spain, in 1983. She received the Telecommunications Engineer degree from the Polytechnic University of Cartagena (UPCT), Murcia, Spain, in 2005, and is currently working toward the Ph.D. degree at the Ecole Nationale Supérieure de Télécommunications (ENST), Paris, France.

Her research interests are in the fields of antenna array signal processing and radio channel parameter estimation.

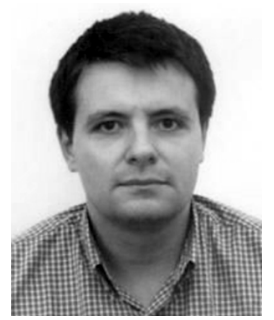

Alejandro Álvarez-Melcón (M'99) was born in Madrid, Spain, in 1965. He received the Telecommunications Engineer degree from the Polytechnic University of Madrid (UPM), Madrid, Spain, in 1991, and the Ph.D. degree in electrical engineering from the Swiss Federal Institute of Technology, Lausanne, Switzerland, in 1998.

In 1988, he joined the Signal, Systems and Radiocommunications Department, UPM, as a Research Student, where he was involved in the design, testing, and measurement of broadband spiral antennas for electromagnetic measurements support (EMS) equipment. From 1991 to 1993, he was with the Radio Frequency Systems Division, European Space Agency (ESA/ESTEC), Noordwijk, The Netherlands, where he was involved in the development of analytical and numerical tools for the study of waveguide discontinuities, planar transmission lines, and microwave filters. From 1993 to 1995, he was with the Space Division, Industry Alcatel Espacio, Madrid, Spain, where he was involved with the ESA and collaborated in several ESA/ESTEC contracts. From 1995 to 1999, he was with the Swiss Federal Institute of Technology, École Polytechnique Fédérale de Lausanne, Lausanne, Switzerland, where he was involved in the field of microstrip antennas and printed circuits for space applications. In 2000, he joined the Technical University of Cartagena, Cartagena, Spain, where he currently develops his teaching and research activities.

Dr. Alvarez-Melcón was the recipient of the Journée Internationales de Nice sur les Antennes (JINA) Best Paper Award for the best contribution to the JINA'98 International Symposium on Antennas, and the Colegio Oficial de Ingenieros de Telecomunicación (COIT/AEIT) Award for the best doctoral thesis in basic information and communication technologies. 